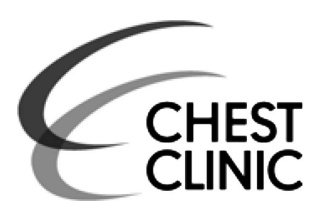
CLINIC

- Additional material is published online only. To view please visit the journal online (http://dx.doi.org/10.1136/ thoraxjnl-2013-204880).

${ }^{1}$ Respiratory Unit, Bambino Gesù Children's Hospital, IRCCS, Rome, Italy 2ENT Department, Bambino Gesù Children's Hospital, IRCCS, Rome, Italy

${ }^{3}$ Department of Imaging, Bambino Gesù Children's Hospital, IRCCS, Rome, Italy ${ }^{4}$ Department of Pediatric Surgery, Bambino Gesù Children's Hospital, IRCCS, Rome, Italy

${ }^{5}$ Department of Pathology, Bambino Gesù Children's Hospital, IRCCS, Rome, Italy ${ }^{6}$ Department of Pediatrics,

"Sapienza" University of Rome, Rome, Italy

\section{Correspondence to} Dr Nicola Ullmann, Respiratory Unit, Bambino Gesù Children's Hospital, IRCCS, Piazza Sant'Onofrio 4, Rome 00100, Italy; nicolaullmann@gmail.com

Received 27 November 2013 Revised 3 December 2013 Accepted 4 December 2013 Published Online First 24 December 2013

\section{CrossMark}

\footnotetext{
To cite: Petreschi $F$, Ullmann N, Bottero S, et al. Thorax 2014;69:396-398.
}

\title{
A young girl with apparent treatment-resistant asthma treated at Bambino Gesù children's hospital
}

\author{
Francesca Petreschi, ${ }^{1}$ Nicola Ullmann, ${ }^{1}$ Sergio Bottero, ${ }^{2}$ Paolo Tomà, ${ }^{3}$ \\ Alessandro Inserra, ${ }^{4}$ Paola Francalanci, ${ }^{5}$ Anna Maria Zicari, ${ }^{6}$ Renato Cutrera ${ }^{1}$
}

Francesca Petreschi (FP): A 9.8 year-old-girl was first admitted in our unit for a persistent bronchospasm, poorly responsive to salbutamol, and progressive onset of respiratory distress after spontaneous expectoration of a bronchial cast (figure 1A). Her clinical history was uneventful until she was 7 years old, when she suffered from two subsequent bouts of pneumonia, but no admission was required. In the following years, she presented few episodes of bronchospasm, clinically diagnosed, with good response to bronchodilator and no limitation on her daily activities. Her family history was positive for allergic disease. On physical examination at her arrival, she appeared quite unwell with persistent wet cough, bilateral respiratory wheeze but normal levels of saturation. The remaining examination was normal.

Renato Cutrera (RC): We have a previously healthy young girl with positive family history for allergies and personal history of few lower respiratory infections and intermittent episodes of wheezing. Patient's history and the spontaneous expectoration of a bronchial cast during an acute episode of bronchospasm, together with her physical examination characterised by intense cough and diffuse wheeze are suggestive of a case of plastic bronchitis (PB), already described as possible complication of allergic asthma. The differential diagnosis, though, should always include more inflammatory diseases of the lung, such as cystic fibrosis, pulmonary infections and acute chest syndrome in sickle-cell anaemia. ${ }^{1}$

Nicola Ullmann (NU): Patient was afebrile; blood exams were normal, including: haemoglobin, creactive protein, immunoglobulins and lipid values. Allergy tests were positive to house dust mites and few pollen, but sweat tests and nasal brushing for ciliary dyskinesia were negative. Spirometry showed mainly an obstructive condition (FEV1: 58\% FEV1/ FVC: 69\%). Normal alveolar capillary diffusion was detected. Chest X-ray showed diffused air trapping with marked and widespread interstitial lung disease. Nocturnal oximetry showed episodes of desaturation and sporadic events with $\mathrm{SpO}_{2}<90 \%$.

$\mathrm{RC}$ : According to the scarce literature based on small number of cases of PB, we know that heart conditions, particularly in post-Fontan surgery, can be associated with bronchial cast formation and often poor prognosis, therefore, we should doublecheck the patient's personal history and examinations on cardiac disease. ${ }^{1}$ Additionally, it would be useful to perform a CT scan and organise a diagnostic bronchoscopy with broncho-alveolar lavage (BAL) for culture.
FP: Further questioning identified no history of cardiac disease or any previous surgical intervention; moreover, the heart examination and the ECG were normal. The patient did not improve with inhaled treatment, and a CT scan was performed showing diffuse interstitial involvement, interlobular and peribronchial septal thickening. She underwent bronchoscopy under general anesthesia: surprisingly, no cast, but a fistula secreting milky material from the left main bronchus (figure $1 \mathrm{~B}$ and see online supplementary video file) was described. The aspiration liquid chemical analysis showed significant high levels of triglyceride, and BAL was negative for cultures.

RC: After an interdisciplinary meeting to discuss CT and bronchoscopy findings, a pulmonary lymphatic disorder with chyloptysis and bronchial cast formation was suspected. Languepin et $a l^{2}$ documented abnormalities in lymphatic drainage with endobronchial leakage contributing to the formation of bronchial casts in three patients with underlying cardiac abnormalities. Chyloptysis, though, can have various aetiologies that need to be taken into account. The most common cause is represented by an increase of systemic or pulmonary venous pressure, usually following heart surgery or cardiac conditions (ie, mitral valve stenosis), that can be ruled out because all cardiac diseases were excluded. As previously published though, in differential diagnoses, we need to consider a possible abnormality of pulmonary lymphatics (pulmonary lymphangiectasia and lymphangiomatosis) and thoracic duct-related disease (trauma, agenesis or obstructive forms). With the suspicion of a lymphatic disorder, it could be useful to perform a lymphoscintigraphy to study the lymphatic system and the lymph drainage.

NU: Lymphoscintigraphy showed normal lymphatic drainage of neck and arms, as well as normal lymphatic drainage of the lower body until the cistern of Pecquet with no visualisation of the liver and portal trunk, suggesting dysplasia of lymphatic return possibly resulting in lung stasis. In order to reduce the portion of the abdominal lymph tributary to the lung, a low-fat diet with medium-chain triglyceride was started in association with medical therapy and physiotherapy. The patient could be soon discharged, but in 1 month, she presented a new onset of respiratory symptoms, chyloptisis, and she was again admitted. On examination, her overall condition had deteriorated considerably, the transcutaneous oxygen saturation was $<88 \%$, chest auscultation showed bilateral wheezing, decreased 
Figure 1 (A) Bronchial cast.

(B) Bronchial fistula secreting milky material under pressure.

(C) CT scan of the thorax

demonstrating left lower lobe opacity and pleural effusion. (D) Lung biopsy compatible with pulmonary lymphangiectasia. (E) CT scan showing chylopericardium.
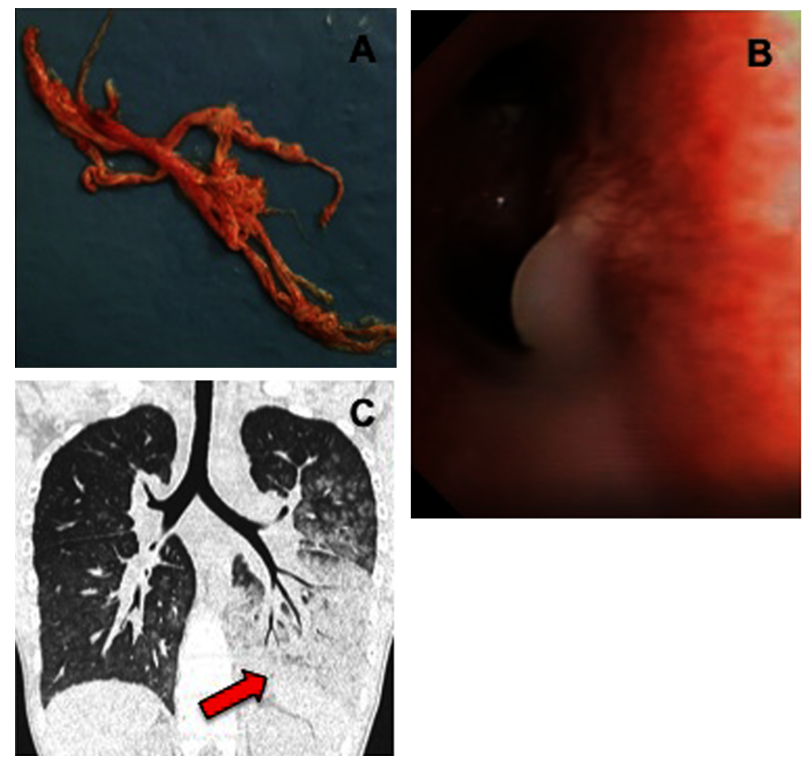
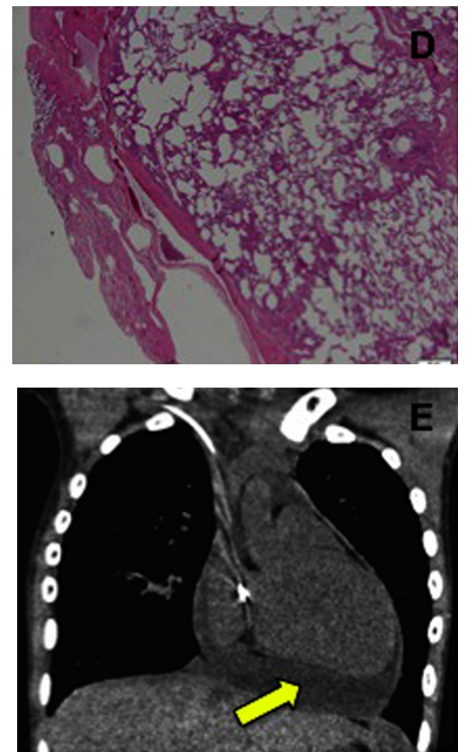

air entry bilaterally, and diffuse crackles. Chest X-ray showed left lower lobe opacity, persistent wide interstitial lung disease and initial pleural effusion. Spirometry showed severe restrictive defect (FEV1 42\%, FEV1/FVC: 93\%). Bronchoscopy revealed many white secretions and a thick cast into the lower bronchus that was removed. The cast consisted of fibrin with few lymphocytes and some eosinophilic inflammatory cells. All cultures remained negative.

RC: Since the lymphoscintigraphy was consistent with a form of lymphatic disorder, possibly a thoracic duct anomaly with lymph stasis in the lung (previously suggested by CT images), we decided to start a supportive treatment and a low-fat diet with medium-chain triglycerides. Unfortunately, the benefit was just partial with rapid formation of another bronchial cast, which was immediately removed with bronchoscopy. ${ }^{3}$ The histology of the bronchial sample would suggest, according to Seear's cast classification, of a combination between a type 2 cast (acellular and more common in children with underlying cardiac defects) and a type 1, because of the partial inflammatory composition, probably secondary to the patient's allergic asthmatic condition. ${ }^{4}$ In literature, it has been hypothesised that a better outcome is possible in patients with type 1 casts with good response to standard anti-inflammatory therapy. ${ }^{1}$ In our patient though, the absence of a clear inflammatory cast's composition, and the poor response to classic asthma treatment suggest a more complex underlying pathological condition responsible of the recurrence of cast formation. Various therapies for bronchial casts have been reported, but the evidence remains anecdotal. ${ }^{3}$ According to fibrin cast histology, a treatment with nebulised tissue plasminogen activator (Alteplase) could be started following the clinical response.

FP: A plasminogen activator inhaled treatment was administered with rapid improvement, but 2 weeks later, her clinical conditions became poorer, oxygen treatment was needed, and she presented a radiological worsening with onset of chylopericardium (figure 1C,E). A total parenteral nutrition, with the aim of completely eliminate fats from diet, was then started, and again a rapid benefit was achieved.

RC: Concerning her repeated chylous effusion and episodes of chyloptisis, despite all medical treatments, and the lack of an available, definitive long-term solution, a surgical approach needs to be discussed in a multidisplinary meeting. As previously seen in the differential diagnosis, we have to consider possible abnormality of pulmonary lymphatics and, before a surgical decision is made, we should better identify the lung pattern and discriminate between pulmonary lymphangiectasia or lymphangiomatosis.

Paola Francalanci-pathologist: The family consented to perform a lung biopsy, and the tissue sample showed a histological condition characterised by normal number but significantly dilated lymphatic vessels (H\&E, D2-40 immunostaining). These findings are suggestive of a pulmonary lymphangectasia (figure1D), but a differentiation between a primary or a secondary condition is not possible.

RC: Late symptom's onset, patient's clinical history and lymphoscintigraphy's findings possibly suggest secondary lymphangectasia determined by lymph stasis as a consequence of a thoracic duct anomaly. This suspect along with the recurrent clinical worsening, due to continuous lymph congestion of the lungs, justify a surgical attempt with a transabdominal ligation of the thoracic duct.

FP: Surgery was performed, and the patient presented a dramatic clinical improvement, medical treatments were gradually discontinued, ECG showed a complete resolution of chylopericardium, FEV1 improved by 33\%, and the young girl was soon discharged with no treatment at all. One year later, her clinical conditions and lung function remains optimal (FEV1: 92\%), no recurrence of cast formation or chyloptisis has been referred, and finally she is back to her usual activities and free diet.

$\mathrm{RC}$ : $\mathrm{PB}$ is an uncommon potentially fatal disease of the airways, often associated with cyanotic heart condition, but unfortunately, only palliative options are available. The pathogenesis of PB is not fully understood, but chyle in bronchial cast, suggests abnormal lymphatic flow. ${ }^{5}$ Our patient presented with intermittent respiratory distress, recurrent chyloptysis and bronchial cast formation. The investigations oriented towards a lymphatic abnormality. We hypothesised that a thoracic-duct dysplasia could have been responsible for an increase of resistance downstream the dysplastic tract, with retrograde flow and consequent formation of a lymphatic leakage into the left main bronchus through a bronchial fistula. The transabdominal duct ligation allowed complete recovery of lung function and the patient's usual activities, without need for any medical treatment. 
To our best knowledge, this is the first case of paediatric PB, without an underlying congenital cardiac disease, which was treated successfully with a thoracic duct ligation. Given further innovative, is the transabdominal duct ligation (vs thoracic), less invasive and effectively safer when the exact location of duct obstruction is uncertain. We believe our case can be a useful example for all forms of $\mathrm{PB}$ with an increased intraductal lymphatic pressure for obstruction or malformation of the thoracic duct.

Acknowledgements We thank Professor Matthias Griese for his expertise and help in the management of this case.

Contributors FP: treated the patient and contributed to the manuscript; NU: conceived the manuscript and treated the patient; SB: performed all bronchoscopies and provided figure and video of secreting fistula; PT: performed all radiological images and contributed to the radiological aspects of discussion; Al: performed the surgical intervention and contributed to the decisional aspects of discussion; PF: reviewed the histopathology and contributed to the pathological aspects of discussion; AMZ: treated the patient initially and contributed to the manuscript; RC: treated the patient, contributed to the manuscript and the clinical aspects of discussion and final decision.

Competing interests None.

Patient consent Obtained.

Ethics approval Obtained.

Provenance and peer review Not commissioned; internally peer reviewed.

\section{REFERENCES}

1 Brogan T, Finn L, Pyskaty J Jr. et al. Plastic bronchitis in children: a case series and review of the medical literature. Pediatr Pulmonol 2002;34:482-7.

2 Languepin J, Scheinmann P, Mahut B, et al. Bronchial casts in children with cardiopathies: the role of pulmonary lymphatic abnormalities. Pediatr Pulmonol 1999:28:329-36.

3 Kruger J, Shpringer C, Picard E, et al. Thoracic air leakage in the presentation of cast bronchitis. Chest 2009;136:615-17.

4 Seear $\mathrm{M}$, Hui $\mathrm{H}$, Magee $\mathrm{F}$, et al. Bronchial casts in children: a proposed classification based on nine cases and a review of the literature. Am J Respir Crit Care Med 1997:155:364-70

5 Ezmigna DR, Morgan WJ, Witte MH, et al. Lymphoscintigraphy in plastic bronchitis, a pediatric case report. Pediatr Pulmonol 2013;48:515-18. 